\title{
Hecke Groups, Dessins d'Enfants, and the Archimedean Solids
}

\author{
Yang-Hui He ${ }^{1,2,3 * t}$ and James Read ${ }^{1 * t}$ \\ ${ }^{1}$ Merton College, University of Oxford, Oxford, UK, ${ }^{2}$ Department of Mathematics, City University London, London, UK, \\ ${ }^{3}$ School of Physics, Nankai University, Tianjin, China
}

\section{OPEN ACCESS}

Edited by:

Cosmas K. Zachos,

Argonne National Laboratory, USA

Reviewed by:

Vishnu Jejjala,

University of the Witwatersrand

South Africa

Andreas Gustavsson,

University of Seoul, South Korea Chryssomalis Chryssomalakos,

Institute for Nuclear Sciences -

UNAM, Mexico

*Correspondence: Yang-Hui He

hey@maths.ox.ac.uk; James Read

james.read@merton.ox.ac.uk

${ }^{\dagger}$ These authors have contributed equally to this work.

Specialty section

This article was submitted to Mathematical Physics, a section of the journal

Frontiers in Physics

Received: 07 October 2015 Accepted: 20 November 2015 Published: 15 December 2015

Citation:

He Y-H and Read J (2015) Hecke Groups, Dessins d'Enfants, and the Archimedean Solids.

Front. Phys. 3:91

doi: 10.3389/fphy.2015.00091
Grothendieck's dessins d'enfants arise with ever-increasing frequency in many areas of twenty-first century mathematical physics. In this paper, we review the connections between dessins and the theory of Hecke groups. Focussing on the restricted class of highly symmetric dessins corresponding to the so-called Archimedean solids, we apply this theory in order to provide a means of computing representatives of the associated conjugacy classes of Hecke subgroups in each case. The aim of this paper is to demonstrate that dessins arising in mathematical physics can point to new and hitherto unexpected directions for further research. In addition, given the particular ubiquity of many of the dessins corresponding to the Archimedean solids, the hope is that the computational results of this paper will prove useful in the further study of these objects in mathematical physics contexts.

Keywords: Hecke groups, dessins d'enfants, Archimedean solids, Platonic solids, Belyi maps

\section{INTRODUCTION}

Grothendieck's dessins d'enfants-bipartite graphs drawn on Riemann surfaces-arise with everincreasing frequency in twenty-first century mathematical physics, appearing in e.g., the study of $\mathcal{N}=2$ supersymmetric gauge theories [1-3], elliptically fibred Calabi-Yaus [4-6], toric conformal field theories $[7,8]$, topological strings $[9,10]$, and elsewhere. Given this growing ubiquity, it is valuable to study both the basic theory of dessins, and the applications of this theory to particularly significant cases. This paper aims to achieve both such goals, by first reviewing the mathematical results which connect dessins d'enfants to the theory of Hecke groups, before applying this work to the dessins for the so-called Archimedean solids. The hope is to provide a compendium of computational results to aid future research featuring these objects.

With the above in mind, let us begin our story by recalling that the Platonic solids are the convex polyhedra with equivalent faces composed of congruent convex regular polygons; these objects have been known and studied for millennia. These solids also appear in mathematical physics: for example, the symmetries of the Platonic solids are related to D-brane orbifold theories [11]. Now, more broadly, another well-known class of convex polyhedra are the Archimedean solids: the semi-regular convex polyhedra composed of two or more types of regular polygons meeting in identical vertices, with no requirement that faces be equivalent. There are three categories of such Archimedean solids: (I) the Platonic solids; (II) two infinite series solutions - the prisms and anti-prisms; and (III) 14 further exceptional cases.

In Magot and Zvonkin [12], a novel approach to the Archimedean solids was taken by interpreting the graphs of these solids as clean dessins d'enfants. By clean, we mean that all the nodes of one of the two possible colors of the bipartite graph have valency two $[13,14]$. Now, the planar graph of any polytope can be interpreted as a clean dessin by inserting a black node into every edge of the graph, and coloring every vertex white. In this way, one can construct dessins for 
all the Archimedean solids. In each case, the underlying Riemann surface is the sphere $\mathbb{C P}^{1}$, and the dessins are drawn in a planar projection.

In a parallel vein, trivalent clean dessins can be associated to conjugacy classes of subgroups of the modular group $\Gamma=$ $\operatorname{PSL}(2, \mathbb{Z})$. (Recall that the modular group $\Gamma \equiv \Gamma(1)$ is the group of linear fractional transformations $z \rightarrow \frac{a z+b}{c z+d}$, with $a, b, c, d \in \mathbb{Z}$ and $a d-b c=1$. The presentation of $\Gamma$ is $\left\langle x, y \mid x^{2}=y^{3}=I\right\rangle$.) To do so, we replace all $n$-valent vertices of the dessin with oriented $n$-gons. This constructs a Schreier coset graph, which displays the permutation action of generators $x$ and $y$ on each coset $G x_{i}$ of $\operatorname{PSL}(2, \mathbb{Z})=\bigcup_{i} G x_{i}, i=1, \ldots, \mu$, where $\mu$ is the index of some subgroup $G$ in PSL $(2, \mathbb{Z})$ [15]. Generalizing, any clean dessin (not necessarily trivalent) can be associated with a conjugacy class of subgroups of a certain so-called Hecke group $H_{n}$, defined as having presentation $\left\langle x, y \mid x^{2}=y^{n}=I\right\rangle$.

Once a Hecke subgroup $G$ has been associated to the dessin in question, further results follow. For example, one can quotient the upper half plane by $G$ to construct the surface $\mathcal{H} / G$. From this, one can construct a Belyi map (in a manner detailed in the main body of this paper), i.e., a holomorphic map to $\mathbb{P}^{1}$ ramified at only $\{0,1, \infty\}$. To each Belyi map there corresponds a unique dessin; precisely the dessin with which we began! Hence, this chain of connections compactifies to a circle.

Returning now to the Archimedean solids, we see that, interpreting these as dessins, we should be able to explore the above circle of connections via explicit computations. Indeed, the Belyi maps associated to these dessins have already been computed in Magot and Zvonkin [12]; therefore, our task is to complete the circle by computing representatives of the conjugacy classes of Hecke subgroups associated to these objects. Not only will this provide an illustration of this aspect of the mathematical theory underlying dessins, but it will also provide a useful resource for further research in this area. Indeed, given that some dessins for the Archimedean solids have have already arisen in areas of mathematical physics (see for example $[3,4,6]$ ), it is not unreasonable to expect that such dessins will continue to manifest themselves in future research.

The structure of this paper is as follows. In Section 2 we present some technical details regarding Hecke groups and dessins d'enfants. We show that it is possible to interpret each clean dessin as the Schreier coset graph for a conjugacy class of subgroups of a certain Hecke group, and review the circle of connections mentioned above. In Section 3, we find the permutation representations for the conjugacy classes of subgroups of Hecke groups corresponding to every Archimedean solid, and provide an algorithm to compute explicit generating sets of matrices for representatives of these conjugacy classes in each case. In Section 4, we close with some conclusions, returning to the specific applications of this work in various subfields of mathematical physics.

\section{DESSINS D'ENFANTS AND HECKE GROUPS}

In this section, we review some essential details regarding both Hecke groups and clean dessins d'enfants. We begin by considering the modular group $\Gamma \cong H_{3}$, as although this is isomorphic to only one particular Hecke group, it is by far the most well-studied, and we shall draw upon the presented results at several points in the ensuing discussion. Subsequently, we discuss Hecke groups more generally, before moving on to consider clean dessins and their associated Belyi maps. Note that the connection between trivalent dessins and the modular group is discussed in Jones and Singerman [16, 17], while the relation between Hecke groups and maps is discussed in e.g., [18]. With these results in hand, we describe how every clean dessin is isomorphic to the Schreier coset graph for a conjugacy class of subgroups of a Hecke group, and how the circle of connections closes via a correspondence between such subgroups and the Belyi maps for the original dessins.

\subsection{The Modular Group}

To begin, let us recall some essential details regarding the modular group $\Gamma$. This is the group of linear fractional transformations $\mathbb{Z} \ni z \rightarrow \frac{a z+b}{c z+d}$, with $a, b, c, d \in \mathbb{Z}$ and $a d-b c=$ 1. It is generated by the transformations $T$ and $S$ defined by:

$$
T(z)=z+1 \quad, \quad S(z)=-1 / z .
$$

The presentation of $\Gamma$ is $\left.\langle S, T| S^{2}=(S T)^{3}=I\right)$, and we will later discuss the presentations of certain modular subgroups. The $2 \times 2$ matrices for $S$ and $T$ are as follows:

$$
T=\left(\begin{array}{ll}
1 & 1 \\
0 & 1
\end{array}\right) \quad, \quad S=\left(\begin{array}{cc}
0 & -1 \\
1 & 0
\end{array}\right) .
$$

Letting $x=S$ and $y=S T$ denote the elements of order 2 and 3 , respectively, we see that $\Gamma$ is the free product of the cyclic groups $C_{2}=\left\langle x \mid x^{2}=I\right\rangle$ and $C_{3}=\left\langle y \mid y^{3}=I\right\rangle$. It follows that $2 \times 2$ matrices for $x$ and $y$ are:

$$
x=\left(\begin{array}{cc}
0 & -1 \\
1 & 0
\end{array}\right) \quad, \quad y=\left(\begin{array}{cc}
0 & -1 \\
1 & 1
\end{array}\right)
$$

With these details in hand, we can consider some important subgroups of $\Gamma$.

\subsubsection{Congruence Modular Subgroups}

The most significant subgroups of $\Gamma$ are the congruence subgroups, defined by having the entries in the generating matrices $S$ and $T$ obeying some modular arithmetic. Some conjugacy classes of congruence subgroups of particular note are the following:

- Principal congruence subgroups:

$$
\Gamma(m):=\{A \in \mathrm{SL}(2 ; \mathbb{Z}) ; A \equiv \pm I \bmod m\} /\{ \pm I\} ;
$$

- Congruence subgroups of level $m$ : subgroups of $\Gamma$ containing $\Gamma(m)$ but not any $\Gamma(n)$ for $n<m$;

- Unipotent matrices:

$$
\Gamma_{1}(m):=\left\{A \in \operatorname{SL}(2 ; \mathbb{Z}) ; A \equiv \pm\left(\begin{array}{ll}
1 & b \\
0 & 1
\end{array}\right) \bmod m\right\} /\{ \pm I\}
$$


- Upper triangular matrices:

$$
\Gamma_{0}(m):=\left\{\left(\begin{array}{ll}
a & b \\
c & d
\end{array}\right) \in \Gamma ; c \equiv 0 \bmod m\right\} /\{ \pm I\}
$$

- The congruence subgroups

$$
\Gamma\left(m ; \frac{m}{d}, \epsilon, \chi\right):=\left\{ \pm\left(\begin{array}{cc}
1+\frac{m}{\epsilon \chi} \alpha & d \beta \\
\frac{m}{\chi} \gamma & 1+\frac{m}{\epsilon \chi} \delta
\end{array}\right) ; \gamma \equiv \alpha \bmod \chi\right\}
$$

for certain choices of $m, d, \epsilon, \chi($ see $[4,6])$.

We note here that:

$$
\Gamma(m) \subseteq \Gamma_{1}(m) \subseteq \Gamma_{0}(m) \subseteq \Gamma
$$

In Section 3 of this paper, we shall remark on the connections between some specific conjugacy classes of congruence modular subgroups and the Archimedean solids.

\subsection{Hecke Groups}

We can now extend our discussion of the modular group $\Gamma \cong \mathrm{H}_{3}$ to the more general Hecke groups $H_{n}$. The Hecke group $H_{n}$ has presentation $\left\langle x, y \mid x^{2}=y^{n}=I\right\rangle$, and is thus the free product of cyclic groups $C_{2}=\left\langle x \mid x^{2}=I\right\rangle$ and $C_{n}=\left\langle y \mid y^{n}=I\right\rangle$. Note that $\Gamma \cong H_{3}$ where $\Gamma$ is the modular group; and that $H_{n}$ is the triangle group $(2, n, \infty) . H_{n}$ is generated by transformations $T$ and $S$ now defined by:

$$
T(z)=z+\lambda_{n} \quad, \quad S(z)=-1 / z
$$

where $\lambda_{n}$ is some real number to be determined. The $2 \times 2$ matrices for these $S$ and $T$ are:

$$
T=\left(\begin{array}{cc}
1 & \lambda_{n} \\
0 & 1
\end{array}\right) \quad, \quad S=\left(\begin{array}{cc}
0 & -1 \\
1 & 0
\end{array}\right)
$$

Letting $x=S$ and $y=S T$ as in our discussion of $\Gamma$, we see that $2 \times 2$ matrices for $x$ and $y$ are:

$$
x=\left(\begin{array}{cc}
0 & -1 \\
1 & 0
\end{array}\right) \quad, \quad y=\left(\begin{array}{ll}
0 & -1 \\
1 & \lambda_{n}
\end{array}\right) .
$$

For $H_{n}$, we clearly have $(S T)^{n}=I$, thereby constraining $\lambda_{n}$ for a given $n$ [19]. Diagonalising $y$ to compute $y^{n}$ explicitly places a constraint which allows for a solution of $\lambda_{n}$; we find the following general expression, as well as some important values for small $n$ :

$$
\lambda_{n}=2 \cos (\pi / n)
$$

\begin{tabular}{|c||c|c|c|c|}
\hline$n$ & 3 & 4 & 5 & 6 \\
\hline$\lambda_{n}$ & 1 & $\sqrt{2}$ & $\frac{1+\sqrt{5}}{2}$ & $\sqrt{3}$ \\
\hline
\end{tabular}

In particular, the $\lambda_{n}$ are algebraic numbers.

\subsubsection{Congruence Subgroups of Hecke Groups}

By way of extension of the above discussion of subgroups of the modular group $\Gamma$, it is useful to consider congruence subgroups of Hecke groups. The Hecke groups are discrete subgroups of $\operatorname{PSL}(2, \mathbb{R})$; in fact, the matrix entries are in $\mathbb{Z}\left[\lambda_{n}\right]$, the extension of the ring of integers by the algebraic number $\lambda_{n}$. Note that:

$$
H_{n} \subset \operatorname{PSL}\left(2, \mathbb{Z}\left[\lambda_{n}\right]\right)
$$

However, unlike the special case of the modular group, this inclusion is strict. With this point in mind, we can define the congruence subgroups of Hecke groups in the following way [19]. Let $I$ be an ideal of $\mathbb{Z}\left[\lambda_{n}\right]$. We then define:

$$
\begin{aligned}
\operatorname{PSL}\left(2, \mathbb{Z}\left[\lambda_{n}\right], I\right)= & \left\{\left(\begin{array}{ll}
a & b \\
c & d
\end{array}\right) \in \operatorname{PSL}\left(2, \mathbb{Z}\left[\lambda_{n}\right]\right) ;\right. \\
& a-1, b, c, d-1 \in I\} .
\end{aligned}
$$

By analogy, we also define:

$$
\operatorname{PSL}^{1}\left(2, \mathbb{Z}\left[\lambda_{n}\right], I\right)=\left\{\left(\begin{array}{ll}
a & b \\
c & d
\end{array}\right) \in \operatorname{PSL}\left(2, \mathbb{Z}\left[\lambda_{n}\right]\right) ; a-1, c, d-1 \in I\right\}
$$

$\operatorname{PSL}^{0}\left(2, \mathbb{Z}\left[\lambda_{n}\right], I\right)=\left\{\left(\begin{array}{ll}a & b \\ c & d\end{array}\right) \in \operatorname{PSL}\left(2, \mathbb{Z}\left[\lambda_{n}\right]\right) ; c \in I\right\}$.

Then we can define the congruence subgroups $H_{n}(m), H_{n}^{1}(m)$, and $H_{n}^{0}(m)$ of $H_{n}$ as follows:

$$
\begin{aligned}
& H_{n}(I)=\operatorname{PSL}\left(2, \mathbb{Z}\left[\lambda_{n}\right], I\right) \cap H_{n} ; \\
& H_{n}^{1}(I)=\operatorname{PSL}^{1}\left(2, \mathbb{Z}\left[\lambda_{n}\right], I\right) \cap H_{n} ; \\
& H_{n}^{0}(I)=\operatorname{PSL}^{0}\left(2, \mathbb{Z}\left[\lambda_{n}\right], I\right) \cap H_{n} .
\end{aligned}
$$

We have:

$$
H_{n}(I) \subseteq H_{n}^{1}(I) \subseteq H_{n}^{0}(I) \subseteq H_{n}
$$

By analogy with our discussion of the modular group, we define congruence subgroups of level $m$ of the Hecke group $H_{n}$ as subgroups of $H_{n}$ containing $H_{n}(m)$ but not any $H_{n}(p)$ for $p<$ $m$ [20]. With these details regarding Hecke groups and their subgroups in hand, we can now consider their connections to clean dessins d'enfants.

\subsection{Dessins d'Enfants and Belyi Maps}

A dessin d'enfant in the sense of Grothendieck is an ordered pair $\langle X, \mathcal{D}\rangle$, where $X$ is an oriented compact topological surface and $\mathcal{D} \subset X$ is a finite graph satisfying the following conditions [13]:

1. $\mathcal{D}$ is connected.

2. $\mathcal{D}$ is bipartite, i.e., consists of only black and white nodes, such that vertices connected by an edge have different colors.

3. $X \backslash \mathcal{D}$ is the union of finitely many topological discs, which we call the faces.

We can interpret any polytope as a dessin by inserting a black node into every edge, and coloring all vertices white. This process 

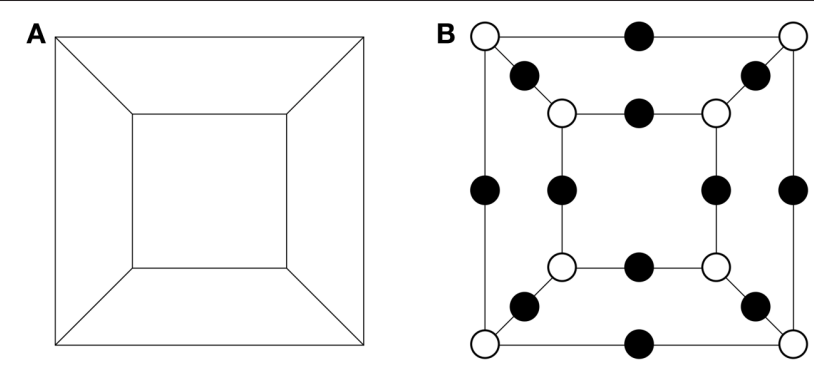

FIGURE 1 | Interpreting the planar graph of the cube as a clean dessin. (A) The planar graph for the cube. (B) The corresponding clean dessin.

of inserting into each edge a bivalent node of a certain color is standard in the study of dessins d'enfants and gives rise to socalled clean dessins, i.e., those for which all the nodes of one of the two possible colors have valency two. An example of this procedure for the cube is shown in Figure 1.

There is a one-to-one correspondence between dessins d'enfants and Belyi maps [13, 16, 17]. A Belyi map is a holomorphic map to $\mathbb{P}^{1}$ ramified at only $\{0,1, \infty\}$, i.e., for which the only points $\tilde{x}$ where $\left.\frac{\mathrm{d}}{\mathrm{d} x} \beta(x)\right|_{\tilde{x}}=0$ are such that $\beta(\tilde{x}) \in$ $\{0,1, \infty\}$. We can associate a Belyi map $\beta(x)$ to a dessin via its ramification indices: the order of vanishing of the Taylor series for $\beta(x)$ at $\tilde{x}$ is the ramification index $r_{\beta(\tilde{x}) \in\{0,1, \infty\}}$ (i) at that $i$ th ramification point $[4,6]$. To draw the dessin from the map, we mark one white node for the $i$ th pre-image of 0 , with $r_{0}(i)$ edges emanating therefrom; similarly, we mark one black node for the $j$ th pre-image of 1 , with $r_{1}(j)$ edges. We connect the nodes with the edges, joining only black with white, such that each face is a polygon with $2 r_{\infty}(k)$ sides [6]. The converse direction (from dessins to Belyi maps) is detailed in e.g., [13], and involves using the dessin to construct a so-called triangle decomposition of the surface $X$ in which it is embedded, before constructing a unique Belyi function from that decomposition. For more information on dessins and Belyi maps, the reader is referred to Girondo and Gonzalez-Diez [13], Schneps [21], Lochak and Schneps [22, 23].

\subsection{Belyi Maps from Hecke Subgroups}

Let $G$ be a torsion-free subgroup of the Hecke group $H_{n}$ of finite index (i.e., a subgroup that contains no element of finite order other than the identity). Then, the compact surface $X_{G}$ is obtained from the surface $\mathcal{H} / G$ (where $\mathcal{H}$ is the upper half plane) by adding finitely many points on the boundary real line of $\mathcal{H}$, called cusps (in mathematical language: one for each $G$ orbit of boundary points at which the stabilizer is non-trivial). For example, choosing $n=3$ and letting $G$ be the full modular group $\Gamma$, there is a single cusp, equivalent to any rational number under $G$, namely, the point at infinity-this is the onepoint compactification of the complex plane into the Riemann sphere. Then (returning to the general case), after carrying out this compactification, the holomorphic projection $\pi: \mathcal{H} / G \rightarrow$ $\mathcal{H} / H_{n} \cong \mathbb{C}$ extends to a Belyi map $\beta: X_{G} \rightarrow \mathbb{P}^{1}$, with each cusp of $X_{G}$ mapped by $\beta$ to the single cusp $\{\infty\}$ of $H_{n}$ which compactifies the plane $\mathcal{H} / H_{n}$ to $\mathbb{C} \cup \infty=\mathbb{P}^{1}$ [24]. In turn, this Belyi map will have a unique associated dessin, as discussed in the previous section.

\subsection{Schreier Coset Graphs}

As mentioned, the Hecke group $H_{n}$ has presentation $\left\langle x, y \mid x^{2}=y^{n}=I\right\rangle$, and is thus the free product of cyclic groups $C_{2}=\left\langle x \mid x^{2}=I\right\rangle$ and $C_{n}=\left\langle y \mid y^{n}=I\right\rangle$. Given the free product structure of $H_{n}$, we see that its Cayley graph is an infinite free $n$-valent tree, but with each node replaced by an oriented $n$-gon. Now, for any finite index subgroup $G$ of $H_{n}$, we can quotient the Cayley graph to arrive at a finite graph by associating nodes to right cosets and edges between cosets which are related by action of a group element. In other words, this graph encodes the permutation representation of $H_{n}$ acting on the right cosets of $G$. This is called a Schreier coset graph, sometimes also referred to in the literature as a Schreier-Cayley coset graph, or simply a coset graph.

It is useful consider the permutations induced by the respective actions of $x$ and $y$ on the cosets of each Hecke subgroup; denote these by $\sigma_{0}$ and $\sigma_{1}$, respectively. We can find a third permutation $\sigma_{\infty}$ by imposing the following condition, thereby constructing a permutation triple $[16,17]$ :

$$
\sigma_{0} \cdot \sigma_{1} \cdot \sigma_{\infty}=1
$$

The permutations $\sigma_{0}, \sigma_{1}$, and $\sigma_{\infty}$ give the permutation representations of $H_{n}$ on the right cosets of each subgroup in question. As elements of the symmetric group, $\sigma_{0}$ and $\sigma_{1}$ can easily be computed from the Schreier coset graphs by following the procedure elaborated in Sebbar [25], i.e., by noting that the doubly directed edges represent an element $x$ of order 2 , while the positively oriented triangles represent an element $y$ of order $n$. Since the graphs are connected, the group generated by $x$ and $y$ is transitive on the vertices. Clearly, $\sigma_{0}$ and $\sigma_{1}$ tell us which vertex of the coset graph is sent to which, i.e., which coset of the Hecke subgroup in question is sent to which by the action of $H_{n}$ on the right cosets of this subgroup.

Now, as detailed in Singerman and Wolfart [26], there is a direct connection between the Schreier coset graphs and the dessins d'enfants for each class of Hecke subgroups: the dessins d'enfants for a certain conjugacy class of subgroups of a Hecke group $H_{n}$ can be constructed from the Schreier coset graphs by replacing each positively oriented $n$-gon with a white node, and inserting a black node into every edge. Conversely, the Schreier coset graphs can be constructed from the dessins by replacing each white node with a positively oriented $n$-gon, and removing the black node from every edge. Crucially, the dessin constructed in this way is the same dessin as that associated to the Belyi map for the surface $X_{G}$ as discussed above, where $G$ is the conjugacy class of Hecke subgroups under consideration [24, 26].

This last result means that our chain of connections between Hecke groups and dessins d'enfants has come full circle: for a given conjugacy class of subgroups $G$ of a Hecke group $H_{n}$, there is an associated (compactified) surface $X_{G}$; in each case there exists a Belyi map $\beta: X_{G} \rightarrow \mathbb{P}^{1}$. Every such Belyi map has a unique associated dessin, which in turn can be translated into a Schreier coset graph for a certain conjugacy class of Hecke subgroups-precisely the $G$ from which we began! 


\section{HECKE SUBGROUPS AND ARCHIMEDEAN SOLIDS}

Having reviewed the theory connecting dessins d'enfants with Hecke subgroups, we can now apply this theory to the particular case of the Archimedean solids. In this section, we first give a precise definition of these geometrical entities. We then identify the conjugacy class of Hecke subgroups corresponding to each Archimedean solid, by giving the permutation representations $\sigma_{0}$ and $\sigma_{1}$ for each such class. Finally, we discuss some interesting aspects of these results.

\subsection{Platonic and Archimedean Solids}

The Platonic solids are the regular, convex polyhedra; they are the tetrahedron, cube, octahedron, dodecahedron and icosahedron. In order to introduce the wider class of Archimedean solids, consider planar graphs without loops, and with vertices of degree $k>2$. Following Magot and Zvonkin [12], let us call the list of numbers $\left(f_{1}, f_{2}, \ldots, f_{k}\right)$, where the $f_{i}$ are the number of edges of the adjacent faces taken in the counter-clockwise direction around the vertex, the type of that vertex. Two such lists are equivalent if one can be obtained from the other by (a) making a cyclic shift and (b) inverting the order of the $f_{i}$. A solid is called Archimedean if the types of all its vertices are equivalent $[12]^{1}$.

We emphasize a subtlety here. One informal way of defining the Archimedean solids is as the semi-regular convex polyhedra composed of two or more types of regular polygons meeting in identical vertices, with no requirement that faces be equivalent. Identical vertices is usually taken to mean that for any two vertices, there must be an isometry of the entire solid that takes one vertex to the other. Sometimes, however, it is only required that the faces that meet at one vertex are related isometrically to the faces that meet at the other. On the former definition, the socalled pseudorhombicuboctahedron, also known as the elongated square gyrobicupola, is not considered an Archimedean solid; on the latter it is. The formal definition of the Archimedean solids above corresponds to the latter definition here; it is this latter definition which which we shall use.

The solids which satisfy the condition for being Archimedean are:

I The five Platonic solids;

II Two infinite series (the prisms and anti-prisms);

III Fourteen exceptional solutions ${ }^{2}$.

All these solids are listed in Table 1. For completeness, we also give the symmetry groups of each of these solids in the column

\footnotetext{
${ }^{1}$ It is worth listing some classical sources on the Archimedean solids. Theses objects were first discussed by Archimedes, in a now lost work to which Pappus refers [27]. In the fifteenth century, the solids were rediscovered by Kepler [28]. Classical geometers who discuss the Archimedean solids include Sommerville [29] and Miller [27].

${ }^{2}$ An interesting aside: It is known that the five Platonic solids correspond to three symmetry groups, which in turn are related to the exceptional Lie algebras $E_{6,7,8}$, and furthermore to Arnold's simple surface singularities with no deformations [30]. It is curious that the next order generalization of the simple singularities, with exactly one deformation modulus, has 14 exceptional cases. We conjecture, therefore, some connection to the exceptional Archimedean solids.
}

labeled "Sym," written in Schönflies notation. Here, we recognize the standard tetrahedral, octahedral and icosahedral groups:

$$
T \simeq A_{4}, \quad O \simeq S_{4}, \quad I \simeq A_{5}
$$

as well as the dihedral group $D_{n}$. The subscripts $d$ and $h$ denote extra symmetries about a horizontal $(h)$ or diagonal $(d)$ plane.

\subsection{Hecke Subgroups for the Archimedean Solids}

As discussed, we can interpret all the Archimedean solids as clean dessins d'enfants by inserting a black node into every edge and coloring every vertex white. By the correspondences detailed in the previous section, we should then be able to associate a class of Hecke subgroups to every Archimedean solid. To achieve this, we begin by converting the dessins for these solids to Schreier coset graphs, reading off the associated permutations $\sigma_{0}$ and $\sigma_{1}$ for the conjugacy class of subgroups of the relevant Hecke group in each case; these results are tabulated in Appendix A. Next, we find a representative of the conjugacy class of subgroups of the relevant Hecke group in each case by inputting the permutations $\sigma_{0}$ and $\sigma_{1}$ into the GAP [31] algorithm presented in Appendix B. This yields a representative of the conjugacy classes of subgroups in each case.

To illustrate this procedure, consider the specific example of the octahedron. Inputting the permutations for the octahedron into the algorithm of Appendix B yields the following generators for a representative of the conjugacy class of subgroups of $\mathrm{H}_{4}$ associated to this solid:

$$
\begin{aligned}
& \left\{\left(\begin{array}{cc}
-1 & 0 \\
3 \sqrt{2} & -1
\end{array}\right),\left(\begin{array}{cc}
1 & -3 \sqrt{2} \\
0 & 1
\end{array}\right),\left(\begin{array}{cc}
-7 & -3 \sqrt{2} \\
6 \sqrt{2} & 5
\end{array}\right),\left(\begin{array}{cc}
-7 & -6 \sqrt{2} \\
3 \sqrt{2} & 5
\end{array}\right),\right. \\
& \left.\left(\begin{array}{cc}
5 & -6 \sqrt{2} \\
3 \sqrt{2} & -7
\end{array}\right),\left(\begin{array}{cc}
5 & -3 \sqrt{2} \\
6 \sqrt{2} & -7
\end{array}\right),\left(\begin{array}{cc}
11 & -15 \sqrt{2} \\
-15 \sqrt{2} & 41
\end{array}\right)\right\} \cdot(19)
\end{aligned}
$$

Note that comparison with our results concerning congruence subgroups of Hecke groups in Section 2.2 reveals that the octahedron corresponds to the principal congruence subgroup $H_{4}$ (3). Repeating this procedure for all Archimedean solids, we find a number of results worthy of comment:

- While the octahedron corresponds to the conjugacy class of Hecke subgroups $\mathrm{H}_{4}(3)$, no other Archimedean solid is readily associated with congruence Hecke subgroups in an analogous manner.

- From Section 2.2, the matrices of $H_{4}$ are of the following two types:

$$
\left(\begin{array}{cc}
a & b \sqrt{2} \\
c \sqrt{2} & d
\end{array}\right) \quad, \quad\left(\begin{array}{cc}
a \sqrt{2} & b \\
c & d \sqrt{2}
\end{array}\right)
$$

The elements of the first type form a subgroup of index 2 in $\mathrm{H}_{4}$; all the subgroups of $\mathrm{H}_{4}$ corresponding to the Archimedean solids turn out to be subgroups of this subgroup.

- We have seen that, interpreted as dessins, the tetrahedron, cube and dodecahedron correspond to the conjugacy classes 
TABLE 1 | The Archimedean solids.

\begin{tabular}{|c|c|c|c|c|c|c|c|}
\hline & Name & $v$ & Vertex type & $\boldsymbol{F}$ & $E$ & Sym & Hecke group \\
\hline 1 & Tetrahedron & 4 & $(3,3,3)$ & 4 & 6 & $T_{d}$ & $H_{3}$ \\
\hline 1 & Cube & 8 & $(4,4,4)$ & 6 & 12 & $O_{h}$ & $H_{3}$ \\
\hline 1 & Dodecahedron & 20 & $(5,5,5)$ & 12 & 30 & $\ln$ & $H_{3}$ \\
\hline 1 & Icosahedron & 12 & $(3,3,3,3,3)$ & 20 & 30 & $\ln$ & $H_{5}$ \\
\hline III & Truncated tetrahedron & 12 & $(3,6,6)$ & 8 & 18 & $T_{d}$ & $H_{3}$ \\
\hline III & Truncated cube & 24 & $(3,8,8)$ & 14 & 36 & $O_{h}$ & $H_{3}$ \\
\hline III & Truncated octahedron & 24 & $(4,6,6)$ & 14 & 36 & $O_{h}$ & $H_{3}$ \\
\hline III & Truncated isocahedron & 60 & $(5,6,6)$ & 32 & 90 & $\ln$ & $H_{3}$ \\
\hline III & Truncated dodecahedron & 60 & $(3,10,10)$ & 32 & 90 & $\ln$ & $H_{3}$ \\
\hline III & Rhombicuboctahedron & 24 & $(3,4,4,4)$ & 26 & 48 & $O_{h}$ & $H_{4}$ \\
\hline III & Rhombicosidodecahedron & 60 & $(3,4,5,4)$ & 62 & 120 & $\ln$ & $H_{4}$ \\
\hline III & Pseudorhombicuboctahedron & 24 & $(3,4,4,4)$ & 26 & 48 & $D_{4 d}$ & $H_{4}$ \\
\hline III & Snub cube & 24 & $(3,3,3,3,4)$ & 38 & 60 & O & $H_{5}$ \\
\hline III & Snub dodecahedron & 60 & $(3,3,3,3,5)$ & 92 & 150 & 1 & $H_{5}$ \\
\hline
\end{tabular}

Type I are the five Platonic solids. Type II are the two infinite families: the prisms and anti-prisms. Type III are the 14 exceptional solids. We record the number of vertices $V$, faces $F$ and edges $E$ in each case. The vertex type is the number of edges of the adjacent faces to the vertex, taken counter-clockwise; the fact that all vertices have the same vertex type is the definition of Archimedean. "Sym" denotes the symmetry group of the solid, given in Schönflies notation. We also indicate the associated Hecke group, determined by the valency of the vertices of each solid.

of subgroups $\Gamma(3), \Gamma(4)$, and $\Gamma(5)$, respectively [6]. In addition to these three arising as the dessins corresponding to certain congruence subgroups of the modular group, the dessins for the 33 conjugacy classes of genus zero, torsionfree congruence subgroups (discussed in detail in $\mathrm{He}$ et al. [6]) reveal that other Archimedean solids also correspond to certain conjugacy classes of congruence subgroups of the modular group. Specifically, the truncated tetrahedron is associated with the modular subgroup $\Gamma_{0}(2) \cap \Gamma$ (3), while the 8-prism is associated with the subgroup $\Gamma(8 ; 2,1,2)$. Given this, the question arises as to whether the other trivalent Archimedean solids can also be associated with congruence subgroups of the modular group. This can be checked in Sage [32] using the permutations $\sigma_{0}$ and $\sigma_{1}$ as input; doing so, we find that the answer to this question is in general negative. As a more specific result, we find that the truncated tetrahedron is the only trivalent, exceptional Archimedean solid which corresponds to a conjugacy class of congruence subgroups of the modular group.

\section{CONCLUSIONS}

In this paper, we have reviewed the connections between dessins d'enfants and Hecke subgroups. We have applied this theory to the case of the Archimedean solids, showing how, through interpretation as clean dessins, each of these geometric objects can be associated with a conjugacy class of subgroups of Hecke groups. In addition to opening up one more area of research into the Archimedean solids, this work is useful from the point of view of mathematical physics, as it can help to shed new light on cases where dessins naturally arise in physical contexts. To take two examples:

1. Certain $\mathcal{N}=2$ supersymmetric gauge theories in four dimensions [33,34] naturally give rise to trivalent dessins [3] (including Archimedean dessins); with the theory of this paper in mind, this suggests an otherwise overlooked connection between these gauge theories and modularity.

2. In He and McKay [4-6], it is shown that certain CalabiYaus give rise to clean, trivalent dessins, including many Archimedean dessins. Again, the theory of this paper therefore suggests a connection between the theory of Hecke groups and these Calabi-Yaus.

The general lessson is the following: given the growing ubiquity of dessins in mathematical physics, it is important to be clear and explicit on the connections of these objects to other areas of mathematics. Hence, it is important to illustrate the theory underlying these connections. It is particularly useful to lay out this theory explicitly in special cases such as that of the Archimedean solids, which we expect (on both inductive grounds and grounds of simplicity) to arise with greatest frequency in physical contexts. 


\section{ACKNOWLEDGMENTS}

YH thanks the Science and Technology Facilities Council, UK, for an Advanced Fellowship and for STFC grant ST/J00037X/1; the Chinese Ministry of Education, for a Chang-Jiang Chair Professorship at NanKai University; the city of Tian-Jin for a

\section{REFERENCES}

1. Ashok S, Cachazo F, DellAquila E. Strebel differentials with integral lengths and Argyres-Douglas singularities. Preprint (2006). arXiv:hep-th/0610080.

2. Ashok S, Cachazo F, DellAquila E. Children's drawings from Seiberg-Witten curves. Commun Number Theory Phys. (2007). 1:237-305. doi: 10.4310/ CNTP.2007.v1.n2.a1

3. He Y-H, Read J. Dessins d'enfants in $\mathcal{N}=2$ generalised quiver theories. $J$ High Energy Phys. (2015) 85:1-29. doi: 10.1007/JHEP08(2015)085

4. He Y-H, McKay J. $\mathcal{N}=2$ gauge theories: congruence subgroups, coset graphs and modular surfaces. J Math Phys. (2013) 54:012301. doi: 10.1063/1.4772976

5. He Y-H, McKay J. Eta products, BPS states and K3 surfaces. J High Energy Phys. (2014) 2014:113. doi: 10.1007/JHEP01(2014)113

6. He Y-H, McKay J, Read J. Modular subgroups, dessins d'enfants and elliptic K3 surfaces. LMS J Comput Math. (2013) 16:271-318. doi: $10.1112 / \mathrm{S} 1461157013000119$

7. Hanany A, He YH, Jejjala V, Pasukonis J, Ramgoolam S, Rodriguez-Gomez D. The beta ansatz: a tale of two complex structures. J High Energy Phys. (2011) 1106:056. doi: 10.1007/JHEP06(2011)056

8. Jejjala V, Ramgoolam S, Rodriguez-Gomez D. Toric CFTs, permutation triples and Belyi Pairs. J High Energy Phys. (2011) 1103:065. doi: 10.1007/JHEP03(2011)065

9. Dumitrescu O, Mulase M, Safnuk B, Sorkin A. The spectral curve of the Eynard-Orantin recursion via the Laplace transform. In: Dzhamay A, Maruno $\mathrm{K}$, Pierce VU, editors. Algebraic and Geometric Aspects of Integrable Systems and Random Matrices, Contemporary Mathematics, Vol. 593. Boston, MA: American Mathematical Society (2013). pp. 263-315.

10. Kazarian M, Zograf P. Virasoro constraints and topological recursion for Grothendieck's dessin counting. Lett Math Phys. (2015). 105:1057-1084. doi: 10.1007/s11005-015-0771-0

11. Hanany A, He YH. Non-abelian finite gauge theories. High Energy Phys. (1999) 9902:013. doi: 10.1088/1126-6708/1999/02/013

12. Magot N, Zvonkin A. Belyi functions for Archimedean solids. Dis Math. (2000) 217:249-271. doi: 10.1016/S0012-365X(99)00266-6

13. Girondo E, Gonzalez-Diez G. Introduction to Compact Riemann Surfaces and Dessins d'Enfants, London Mathematical Society Student Texts, Vol. 79. Cambridge, UK: Cambridge University Press (2012).

14. Zvonkin A. Functional composition is a generalized symmetry. Symmetry (2011) 22:391-426.

15. McKay J, Sebbar A. J-Invariants of arithmetic semistable elliptic surfaces and graphs. In: Proceedings on Moonshine and Related Topics. Montréal, QC (2001). pp. 119-30.

16. Jones GA, Singerman D. Theory of maps on orientable surfaces. Proc Lond Math Soc. (1978) 3-37:273-307. doi: 10.1112/plms/s3-37.2.273

17. Jones GA, Singerman D. Belyi functions, hypermaps and Galois groups. Bull Lond Math Soc. (1996) 28:561-90. doi: 10.1112/blms/28.6.561

18. Cangül I, Singerman D. Normal subgroups of Hecke groups and regular maps. Math Proc Camb Philos Soc. (1998) 123:59-74. doi: $10.1017 / \mathrm{s} 0305004197002004$

19. Ivrissimtzis I, Singerman D. Regular maps and principal congruence subgroups of Hecke groups. Eur J Comb. (2005) 26:437-456. doi: 10.1016/j.ejc.2004.01.010
Qian-Ren Scholarship; the US NSF for grant CCF-1048082; as well as City University, London; the Department of Theoretical Physics, Oxford; and Merton College, Oxford, for their enduring support. JR is supported by an AHRC studentship at the University of Oxford, and is also indebted to Merton College for their support.

20. Ikikardes S, Sahin R, Cangul IN. Principal congruence subgroups of the Hecke groups and related results. Bull Braz Math Soc. (2009) 40:479-494. doi: 10.1007/s00574-009-0023-y

21. Schneps L, (ed.). The Grothendieck Theory of Dessins D'enfants. London Mathematical Society Lecture Note Series, Vol. 200. Cambridge, UK: Cambridge University Press (1994).

22. Lochak P, Schneps L, (eds.). Geometric Galois Actions 1. London Mathematical Society Lecture Note Series, Vol. 242. Cambridge, UK: Cambridge University Press (1997).

23. Lochak P, Schneps L, (eds.). Geometric Galois Actions 2. London Mathematical Society Lecture Note Series, Vol. 243. Cambridge, UK: Cambridge University Press (1997).

24. Harvey W. Teichmüller spaces, triangle groups and Grothendieck dessins. In: Papadopoulos A, editor. Handbook of Teichmüller Theory, Vol. 1. Zürich: European Mathematical Society (2007). pp. 249-292.

25. Sebbar A. Modular subgroups, forms, curves and surfaces. Can Math Bull. (2002) 45:294-308. doi: 10.4153/CMB-2002-033-1

26. Singerman D, Wolfart J. Cayley graphs, Cori hypermaps, and dessins d'enfants. ARS Math Contemp. (2008) 1:144-53.

27. Grünbaum B. An enduring error. Elem Math. (2009) 64:89-101. doi: 10.4171/EM/120

28. Field J. Rediscovering the Archimedean polyhedra: Piero della Francesca, Luca Pacioli, Leonardo da Vinci, Albrecht Dürer, Daniele Barbaro, and Johannes Kepler. Arch Hist Exact Sci. (1997) 50. doi: 10.1007/BF 00374595

29. Sommerville DMY. Semi-regular networks of the plane in absolute geometry. Trans R Soc Edinb. (1906) 41:725-747. doi: 10.1017/S00804568 00035560

30. Brieskorn E, Pratoussevitch A, Rothenhäusler F. The combinatorial geometry of singularities and Arnold's series E, Z, Q. Mosc Math J. (2003) 3:273-333.

31. The GAP Group. GAP - Groups, Algorithms, and Programming, Version 4.5.6. (2012). Available online at: http://www.gap-system.org

32. Sage. Sage - Version 5.3. (2012). Available online at: http://www.sagemath.org

33. Gaiotto D. $\mathcal{N}=2$ dualities. J High Energy Phys. (2012) 34:8. doi: 10.1007/JHEP08(2012)034

34. Tachikawa Y. $\mathcal{N}=2$ supersymmetric dynamics for pedestrians. Lect. Notes Phys. (2014) 890. doi: 10.1007/978-3-319-08822-8

Conflict of Interest Statement: The authors declare that the research was conducted in the absence of any commercial or financial relationships that could be construed as a potential conflict of interest.

The Reviewer Vishnu Jejjala, has previously published with an author of this manuscript, Dr He; the Editorial Office and Chief Editor confirm the review was carried out to the highest ethical standards.

Copyright (C) 2015 He and Read. This is an open-access article distributed under the terms of the Creative Commons Attribution License (CC BY). The use, distribution or reproduction in other forums is permitted, provided the original author (s) or licensor are credited and that the original publication in this journal is cited, in accordance with accepted academic practice. No use, distribution or reproduction is permitted which does not comply with these terms. 


\section{APPENDIX}

\section{A. PERMUTATIONS FOR THE ARCHIMEDEAN SOLIDS}

In this appendix, we present the permutations $\sigma_{0}$ and $\sigma_{1}$ corresponding to the conjugacy classes of Hecke subgroups for each of the Archimedean solids. Although it is in principle easy to read these permutations off from the Schreier coset graphs in the manner detailed in Section 2.5, the procedure is extremely time-consuming, so it is worth presenting the results in full here. The method for computing explicit generators for a representative of each class of subgroups is detailed in the following appendix.

As a technical point, note that we have just presented the permutations $\sigma_{0}$ in each case. The permutations $\sigma_{1}$ can be written down by populating $2 E / n \quad n$-tuples with numerals in ascending order from 1 to $2 E$, where $E$ is the number of edges of the solid, and $H_{n}$ is the associated Hecke group as given in Table 1. So, for example, the permutations $\sigma_{1}$ for the octahedron are: $(1,2,3,4)(5$, $6,7,8)(9,10,11,12)(13,14,15,16)(17,18,19,20)$ $(21,22,23,24)$.

\section{A.1. Platonic Solids}

Interpreted as clean dessins d'enfants, the tetrahedron, cube, and dodecahedron correspond to the conjugacy classes of principal congruence subgroups $\Gamma$ (3), $\Gamma$ (4), and $\Gamma$ (5), respectively [6]. In addition, from Table 1 we can see that the remaining two Platonic solids-the octahedron and the icosahedron-correspond to conjugacy classes of subgroups of $H_{4}$ and $H_{5}$, respectively ${ }^{3}$. The permutations $\sigma_{0}$ for these classes are:

Octahedron: $(1,5)(6,11)(4,12)(2,13)(3,23)(8,14)(7,18)(10$, 19) $(9,22)(16,24)(15,17)(20,21)$

Icosahedron: $(1,6)(10,11)(5,15)(2,21)(3,16)(4,43)(7,22)$ $(8,27)(9,33)(12,34)(13,37)(14,42)(17,25)(23,26)(28,32)$ $(35,36)(38,41)(20,44)(18,46)(24,47)(30,48)(29,52)(31,53)$ $(40,54)(39,57)(45,58)(19,59)(50,60)(55,56)(49,51)$

\section{A.2. Prisms and Antiprisms}

The prisms and antiprisms form infinite series of Archimedean solids. Since all prisms are trivalent, all correspond to a conjugacy class of subgroups of $\Gamma$; since all antiprisms are 4-valent, all correspond to a conjugacy class of subgroups of $H_{4}$. We can construct general expressions for the permutations $\sigma_{0}$ and $\sigma_{1}$ for the prisms and antiprisms. These expressions can be used to find the permutations $\sigma_{0}$ and $\sigma_{1}$ for any particular (anti)prism of interest.

${ }^{3}$ Of course, one should note that the octahedron and icosahedron could also be defined as subgroups of the modular group $\Gamma$, as their duals are trivalent.

\section{Prisms:}

Call the $n$-gon faced prism the $n$-prism. The $n$-prism has the following permutations $\sigma_{0}$ and $\sigma_{1}$ :

$$
\begin{aligned}
& \sigma_{0}:(3 n-2,3)(3 n-1,6 n-2)(6 n-1,3 n+3) \\
& \cdot \prod_{i=0}^{n-2}(3 i+1,3 i+6)(3 i+2,3 n+3 i+1) \\
& \quad(3 n+3 i+2,3 n+3 i+6) \\
& \sigma_{1}: \prod_{i=0}^{n+2}(3 i+1,3 i+2,3 i+3) .
\end{aligned}
$$

\section{Antiprisms:}

Call the $n$-gon faced anti-prism the $n$-antiprism. The $n$-antiprism has the following permutations $\sigma_{0}$ and $\sigma_{1}$ :

$$
\begin{aligned}
\sigma_{0}: & (4 n-3,4)(4 n-2,8 n-2)(3,8 n-1)(4 n+1,8 n) \\
& \cdot \prod_{i=0}^{n-2}(4 i+1,4 i+8)(4 i+2,4 n+4 i+2) \\
& (4 i+7,4 n+4 i+3)(4 n+4 i+4,4 n+4 i+5) \\
\sigma_{1}: & \prod_{i=0}^{n+2}(4 i+1,4 i+2,4 i+3,4 i+4) .
\end{aligned}
$$

\section{A.3. Exceptional Archimedean Solids}

In addition to the Platonic solids and the prisms and antiprisms, there remain 14 exceptional Archimedean solids, as given in Table 1. Here we give the permutations $\sigma_{0}$ for each corresponding class of Hecke subgroups.

Truncated tetrahedron: $(1,32)(3,4)(2,7)(5,9)(6,24)(8,10)$ $(11,13)(12,18)(15,16)(17,19)(14,28)(29,31)(33,34)(30,36)$ $(26,35)(20,25)(23,27)(21,22)$

Truncated cube: $(3,4)(2,7)(5,9)(60,61)(63,55)(57,58)(20$, 22) $(21,27)(24,25)(11,13)(12,18)(15,16)(39,45)(43,42)(38$, 40) $(30,31)(29,35)(32,34)(66,72)(65,68)(67,70)(48,49)(47$, 53) $(50,52)(1,59)(62,64)(69,54)(51,6)(41,46)(8,10)(56,23)$ $(36,71)(33,37)(17,44)(14,19)(26,28)$

Truncated octahedron: $(1,11)(2,4)(5,8)(9,10)(12,13)(3,36)$ $(6,56)(7,65)(35,26)(32,34)(29,31)(27,28)(25,17)(16,14)$ $(15,24)(21,22)(18,19)(23,68)(70,69)(66,67)(62,64)(63,72)$ $(59,61)(57,58)(51,60)(50,52)(54,55)(49,48)(45,71)(20,42)$ $(30,37)(39,40)(38,47)(44,46)(41,43)(33,53)$

Truncated icosahedron: $(1,14)(11,13)(8,10)(5,7)(2,4)(3,16)$ $(15,28)(12,26)(9,22)(6,20)(19,35)(32,34)(17,31)(18,58)$ $(56,60)(29,55)(30,53)(50,52)(27,49)(25,47)(45,46)(44,23)$ $(24,41)(38,40)(21,37)(36,73)(33,64)(59,61)(57,110)(54$, 107) $(51,98)(48,95)(43,86)(42,83)(39,77)(71,75)(70,68)(65$, 67) $(66,62)(63,116)(115,113)(111,112)(108,109)(106,104)$ $(101,103)(100,99)(96,97)(92,94)(89,91)(87,88)(84,85)(80$, $82)(119,79)(78,118)(74,76)(120,122)(72,164)(69,158)(117$, 155) $(114,149)(146,105)(140,102)(93,137)(131,90)(128,81)$ 
$(163,161)(159,160)(156,157)(152,154)(150,151)(147,148)$ $(143,145)(141,142)(138,139)(134,136)(132,133)(129,130)$ $(125,127)(123,124)(121,165)(162,166)(153,179)(144,176)$ $(135,173)(126,170)(167,169)(171,172)(174,175)(177,178)$ $(168,180)$

Truncated dodecahedron: $(1,29)(3,32)(30,31)(27,44)(23,25)$ $(24,43)(21,41)(18,40)(17,19)(11,13)(15,39)(12,38)(5,7)$ $(9,35)(6,34)(2,4)(26,28)(22,20)(14,16)(8,10)(36,62)(33$, 46) $(45,107)(42,92)(37,77)(61,59)(60,66)(63,64)(47,49)$ $(51,120)(48,119)(108,109)(111,105)(104,106)(90,96)(93$, 94) $(89,91)(74,76)(78,79)(75,81)(58,56)(50,52)(55,53)(54$, 122) $(57,123)(121,136)(117,118)(112,110)(116,113)(115$, 135) $(114,134)(133,149)(101,103)(95,97)(98,100)(102,131)$ $(99,130)(132,146)(86,88)(80,82)(83,85)(87,128)(84,127)$ $(129,142)(71,73)(65,67)(68,70)(72,126)(69,125)(124,139)$ $(137,154)(156,153)(152,138)(151,180)(179,150)(148,176)$ $(177,178)(174,175)(173,147)(170,145)(171,172)(168,169)$ $(165,166)(164,144)(143,167)(162,163)(159,160)(141,158)$ $(140,161)(155,157)$

Truncated cuboctahedron: $(1,23)(24,20)(21,17)(18,14)(15$, 12) $(10,9)(7,6)(4,3)(2,25)(22,29)(19,32)(16,35)(13,38)$ $(11,41)(8,44)(5,46)(26,28)(30,55)(56,58)(31,59)(33,34)$ $(36,62)(63,64)(65,37)(39,40)(42,67)(68,70)(43,71)(45$, 48) $(47,49)(50,52)(27,53)(54,77)(51,73)(76,74)(78,79)(75$, 119) $(57,86)(60,89)(88,87)(85,83)(90,91)(61,98)(66,101)$ $(100,99)(95,97)(102,103)(72,113)(69,110)(111,112)(114$, $115)(107,109)(80,82)(84,128)(126,127)(81,125)(129,130)$ $(132,133)(96,134)(92,94)(93,131)(135,136)(137,105)(104$, 106) $(108,140)(138,139)(141,142)(117,143)(123,144)(120$, 121) $(116,118)(122,124)$

Truncated icosidodecahedron: $(1,29)(26,28)(23,25)(20,22)$ $(17,19)(15,16)(11,14)(8,10)(5,7)(2,4)(3,31)(30,59)(27$, 56) $(24,52)(21,50)(18,47)(13,44)(12,41)(9,38)(6,34)(33$, 60) $(58,89)(88,86)(85,57)(53,55)(83,54)(80,82)(79,51)$ $(48,49)(46,77)(75,76)(45,74)(42,43)(40,71)(68,70)(39,67)$ $(35,37)(36,65)(64,62)(61,32)(90,119)(87,116)(84,113)(81$, 109) $(78,107)(73,104)(72,101)(69,98)(66,95)(63,91)(93$, 121) $(123,179)(178,176)(175,174)(173,120)(118,117)(115$, 170) $(169,167)(166,164)(163,162)(161,114)(112,110)(111$, $158)(155,157)(152,154)(149,151)(108,148)(105,106)(103$, $146)(143,145)(140,142)(138,139)(102,137)(99,100)(97,134)$ $(131,133)(128,130)(125,127)(96,124)(92,94)(122,181)(180$, 269) $(177,260)(257,172)(171,254)(168,251)(165,242)(160$, 239) $(159,236)(156,233)(153,224)(150,221)(147,218)(144$, 215) $(141,206)(136,203)(135,200)(132,197)(129,188)(126$, 185) $(184,182)(183,270)(268,266)(265,262)(264,261)(259$, 258) $(256,255)(252,253)(248,250)(247,245)(244,243)(241$, 240) $(237,238)(234,235)(230,232)(227,229)(226,225)(222$, 223) $(219,220)(216,217)(214,212)(209,211)(207,208)(204$, 205) $(202,201)(199,198)(196,194)(191,193)(189,190)(186$, 187) $(267,271)(263,323)(249,314)(246,311)(231,302)(228$, 299) $(290,213)(210,287)(195,278)(192,274)(276,329)(328$, 326) $(325,272)(273,324)(322,320)(319,317)(316,315)(313$, 312) $(308,310)(307,305)(304,303)(300,301)(296,298)(293$,
295) $(291,292)(288,289)(284,286)(281,283)(279,280)(275$, 277) $(330,332)(327,359)(321,356)(353,318)(350,309)(347$, $306)(344,297)(294,341)(285,338)(282,335)(333,334)(331$, $360)(358,357)(355,354)(351,352)(348,349)(345,346)(342$, 343) $(339,340)(337,336)$

Cuboctahedron: $(1,10)(11,14)(15,8)(4,5)(2,21)(3,17)(20$, 6) $(7,31)(30,16)(27,13)(22,9)(12,26)(25,39)(28,42)(43,29)$ $(32,46)(47,19)(18,33)(24,34)(23,38)(35,37)(40,41)(44,45)$ $(36,48)$

Icosidodecahedron: $(1,9)(10,17)(18,26)(27,34)(35,4)(3,37)$ $(2,5)(6,12)(11,13)(14,20)(19,21)(22,25)(28,29)(30,33)$ $(36,40)(38,41)(44,79)(39,78)(8,45)(7,49)(46,52)(16,54)$ $(55,57)(15,58)(24,63)(64,65)(23,66)(32,70)(31,74)(71,73)$ $(75,77)(80,110)(76,109)(42,48)(47,82)(81,43)(50,53)(56$, $86)(51,85)(60,93)(59,62)(61,94)(67,69)(72,102)(101,68)$ $(89,96)(95,100)(90,99)(98,107)(103,108)(97,104)(105,112)$ $(111,116)(106,115)(84,113)(83,117)(114,120)(88,118)(87$, 92) $(91,119)$

Rhombicuboctahedron: $(1,5)(6,9)(10,13)(4,14)(2,17)(3,30)$ $(20,31)(8,33)(7,38)(34,37)(12,54)(11,59)(55,58)(15,70)$ $(67,69)(16,66)(18,36)(35,48)(21,47)(19,24)(40,41)(39,53)$ $(50,56)(42,49)(57,64)(61,79)(68,80)(65,60)(72,76)(28,73)$ $(25,32)(29,71)(22,82)(27,81)(23,26)(44,45)(46,86)(43,87)$ $(51,63)(62,91)(52,90)(75,77)(74,95)(78,94)(84,96)(83,85)$ $(88,89)(92,93)$

Rhombicosidodecahedron: $(1,19)(4,5)(8,12)(11,16)(15,20)$ $(2,21)(3,28)(6,29)(7,36)(9,40)(10,44)(13,47)(14,52)(17$, 56) $(18,237)(240,22)(24,25)(27,30)(32,33)(35,37)(39,41)$ $(43,48)(46,49)(51,53)(55,238)(54,116)(57,239)(23,68)(26$, $72)(31,80)(34,84)(38,92)(42,96)(45,104)(50,108)(115,58)$ $(60,61)(64,65)(67,69)(71,73)(76,77)(79,81)(83,85)(88$, 89) $(91,93)(95,97)(100,101)(103,105)(107,109)(112,113)$ $(114,117)(175,118)(62,124)(63,128)(66,132)(70,131)(74$, 136) $(75,140)(78,144)(82,143)(86,148)(87,152)(90,156)(94$, 155) $(98,160)(99,164)(102,168)(106,167)(110,172)(111,176)$ $(119,121)(123,125)(127,129)(130,133)(135,137)(139,141)$ $(142,145)(147,149)(151,153)(154,157)(159,161)(163,165)$ $(166,169)(171,173)(59,120)(122,184)(126,188)(134,192)$ $(138,196)(146,200)(150,204)(158,208)(162,212)(170,213)$ $(174,177)(183,185)(187,189)(191,193)(195,197)(199,201)$ $(203,205)(207,209)(211,214)(178,216)(180,181)(182,224)$ $(186,223)(190,228)(194,227)(198,232)(202,231)(206,236)$ $(210,235)(215,218)(179,217)(222,225)(226,229)(230,233)$ $(234,219)(220,221)$

Pseudorhombicuboctahedron: $(1,5)(6,10)(11,14)(4,15)(2$, 17) $(3,46)(20,47)(8,21)(7,26)(22,25)(9,30)(12,35)(31,34)$ $(13,38)(16,43)(39,42)(18,24)(23,53)(50,56)(19,49)(28,58)$ $(59,61)(32,62)(27,29)(33,65)(66,70)(40,71)(36,37)(44,45)$ $(48,80)(76,79)(41,75)(52,77)(51,81)(84,96)(78,95)(55,82)$ $(83,88)(60,85)(54,57)(86,64)(63,68)(67,91)(87,90)(89,93)$ $(73,94)(72,74)(69,92)$ 
Snub cube: $(1,18)(13,17)(8,12)(2,7)(5,22)(4,60)(3,53)(21$, 19) $(20,26)(16,35)(34,14)(40,15)(45,11)(9,44)(10,49)(6$, 54) $(52,56)(59,23)(25,27)(30,31)(33,36)(39,41)(43,50)$ $(48,55)(51,73)(57,72)(58,67)(24,61)(65,28)(29,97)(32$, 93) $(37,92)(38,87)(42,84)(46,83)(47,78)(71,68)(66,62)(64$, 98) $(96,94)(88,91)(85,86)(79,82)(74,77)(75,108)(69,107)$ $(70,103)(63,102)(99,101)(100,119)(118,95)(89,117)(90$, $114)(81,113)(80,112)(76,109)(104,106)(105,120)(115,116)$ $(110,111)$

Snub dodecahedron: $(1,7)(8,12)(13,16)(17,21)(5,22)(2,37)$ $(3,32)(4,27)(6,38)(10,42)(9,47)(11,48)(15,52)(14,57)$ $(20,58)(19,62)(18,67)(25,68)(24,72)(26,23)(28,31)(33$, 36) $(39,41)(43,46)(49,51)(53,56)(59,61)(63,66)(69,71)$ $(30,73)(29,76)(35,87)(34,92)(40,93)(45,102)(44,107)(50$, 108) $(55,117)(54,122)(60,123)(65,132)(64,137)(70,138)(75$, 147) $(74,80)(77,81)(82,86)(88,91)(94,96)(97,101)(103,106)$ $(109,111)(112,116)(118,121)(124,126)(127,131)(133,136)$ $(139,141)(142,146)(79,148)(149,182)(78,183)(85,184)(84$, 187) $(83,192)(90,193)(89,197)(95,198)(100,199)(99,202)$ $(98,206)(105,207)(104,212)(110,213)(115,214)(114,217)$ $(113,223)(120,224)(119,152)(125,153)(130,154)(129,157)$ $(128,162)(135,163)(134,167)(140,168)(145,169)(144,172)$ $(143,177)(150,178)(200,201)(203,210)(208,211)(215,216)$ $(218,222)(225,151)(155,156)(158,161)(164,166)(170,171)$ $(173,176)(179,181)(185,186)(188,191)(194,196)(195,258)$ $(205,262)(204,267)(209,268)(220,272)(219,230)(221,226)$

$(160,232)(159,237)(165,238)(175,242)(174,247)(180,248)$
$(190,252)(189,257)(259,261)(263,266)(269,271)(273,229)$

$(227,231)(233,236)(239,241)(243,246)(249,251)(253,256)$

$(260,293)(265,294)(264,297)(270,298)(275,299)(274,280)$

$(228,276)(235,277)(234,282)(240,283)(284,245)(287,244)$

$(288,250)(289,255)(292,254)(295,296)(300,279)(278,281)$ $(285,286)(290,291)$

\section{B. ALGORITHM FOR COMPUTING GENERATORS FOR HECKE SUBGROUPS}

We can find the generators for a representative of all the conjugacy classes of subgroups of interest using $\operatorname{GAP}[6,31]$. First, we use the permutation data $\sigma_{0}, \sigma_{1}$ obtained from each of the Schreier coset graphs (in turn obtained from each of the dessins) to find the group homomorphism by images between the relevant Hecke group and a representative of the conjugacy class of subgroups of interest. We then use this to define the representative in question. Finally, we use the GAP command IsomorphismFpGroup(G), which returns an isomorphism from the given representative to a finitely presented group isomorphic to that representative. This function first chooses a set of generators of the representative and then computes a presentation in terms of these generators.

To give an example, consider the clean dessin for the octahedron. We can find a set of generators as $2 \times 2$ matrices for a representative of the associated class of subgroups by implementing the following code in GAP:

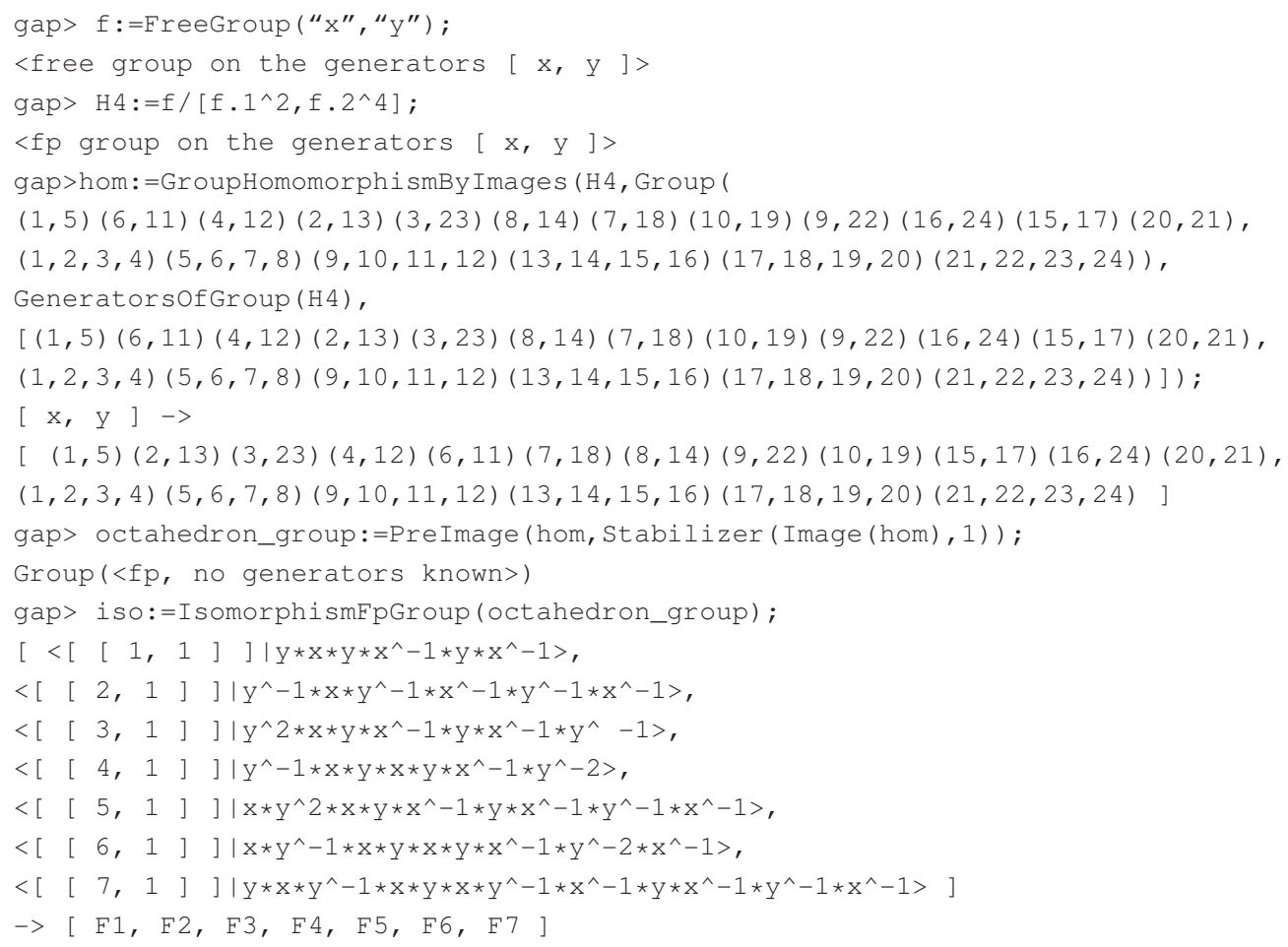


We define the relevant Hecke group (here $H_{4}$ ) in the third line. Then, the only input in each case is the permutation data $\sigma_{0}, \sigma_{1}$. Once the output has been obtained, we see the generators (here seven: $[F 1, F 2, F 3, F 4, F 5, F 6, F 7])$ in the final line, as functions of the $x$ and $y$ for the Hecke group in question. Now, returning to the explicit matrices for these $x$ and $y$ presented in Section 2.2, the only thing left to do is to multiply together the matrices and their inverses as indicated. This will produce the generators for each representative, as required. 